\title{
Design and Validation of Ethernet Network Interface Card using Simevents Technique
}

\author{
Mohammed Younis, Ph.D \\ Electrical Department \\ College of Engineering \\ University of Mosul/IRAQ
}

\author{
A. I. A. Jabbar, Ph.D \\ Electrical Department \\ College of Engineering \\ University of Mosul/IRAQ
}

\begin{abstract}
Simevent proved to be efficient and flexible tool in the design and simulation of computer networks. Ethernet Network Interface Card (NIC) includes Logical Link Controller (LLC), Medium Access Controller (MAC) and physical layer. It is designed using simevent tools (blocks), the units of the NIC can be modified and adjusted separately, and as a matter of check, a network of Ethernet type using simevent blocks is designed based on the proposed NIC then its performance is compared with a similar Ethernet (based on OPNET software), the similarity of the two networks is proved through the simulation results of throughput and delay performances.
\end{abstract}

It is possible to conclude that simulation with simevent tools push the design of network to behave like actual networks and as a consequence there is a possibility to modify the parameters of the different protocols easily.

\section{General Terms}

Ethernet Network Interface Card.

\section{Keywords}

Simevent tools, OPNET, Ethernet, MAC, Sub Layer, NIC

\section{INTRODUCTION}

Recent LANs operate at relatively high speeds (Mbps range and upwards).

The software which controls the transfer of messages among the devices on the LAN must deal with the problems of sharing the common resources of the networks without conflict or corruption of data. Since many users can access the network at the same time, some rules must be established on which devices can access the network, these rules are considered under what is called media access control (MAC) [1].

Network interface card (NIC) of a computer in a network must provide the proper signals and follows a given protocol to access the network physical medium (i.e. MAC protocol) such that maximum throughput can be achieved. Listening to the shared channel and providing the suitable path formatting and signal functions are related to the NIC responsibility.

On the other hand, it is worth to mention that High speed LANs which usually covers a restricted geographical area (For example10Gigabit Ethernet) has a completely different NIC performance technique.

\section{TYPICAL NETWORK INTERFACE CARD}

A typical Ethernet NIC block diagram is shown in Figure (1), according to the TCP/IP model, any data generated at the application layer must pass through other layers until it reaches the NIC which is responsible for inserting it into the common channel $[3,4]$.

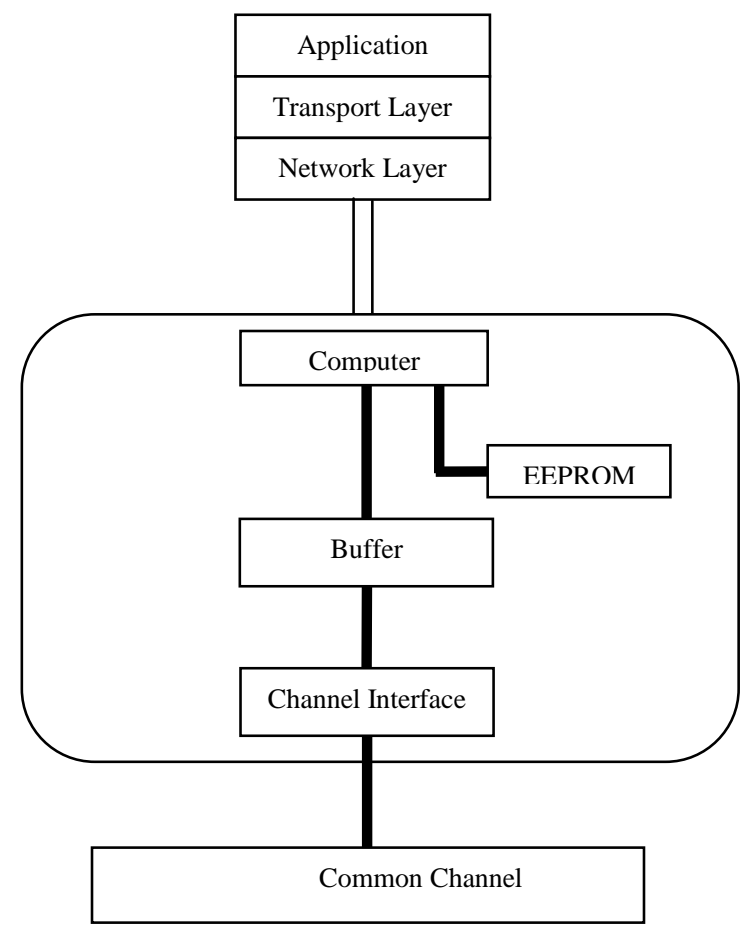

Figure (1) a typical NIC block diagram

It is something known that the functions of data link layer (which includes LLC and MAC) are included in computer interface unit, a buffer is introduced between the computer and channel interfaces units to manipulate the different arrival and departure data rates. Channel interface unit is responsible about the generation of the proper signals and applying the MAC protocol. Finally all control commands are stored in EEPROM.

The function of the Ethernet MAC protocol which is called carrier sense multiple access with collision detection (CSMA/CD) is shown in Figure (2).

A computer according to CSMA/CD protocol must apply the following algorithm:

1- If the channel is idle, transmit the data.

2- If the channel is busy, wait until the channel becomes idle.

3- If two PCs send at the same time or with the propagation delay time, collision will occur.

4- If a host detects a collision during the beginning of a transmission, it must be immediately aborted. 
5- After a back off time, a host that wishing to transmit must attempt a new transmission according to a specific back off algorithm.

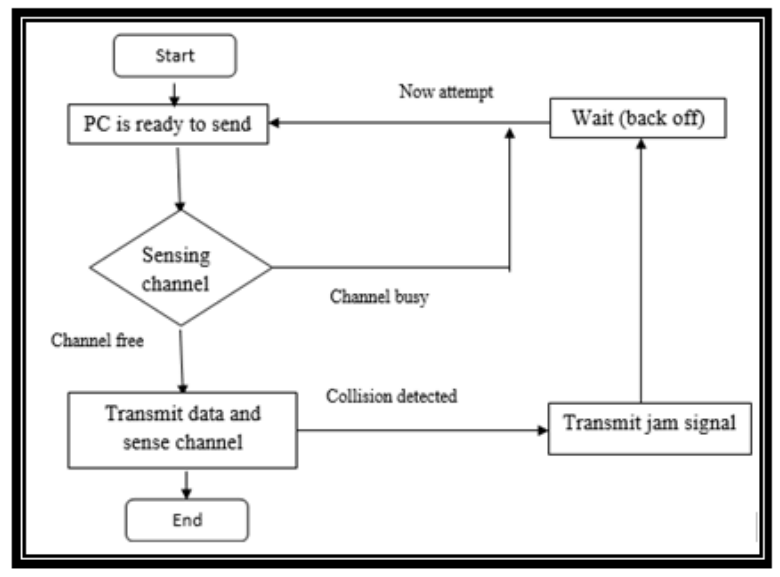

Figure (2) Flow chart of the CSMA/CD protocol

\section{ANALYSIS OF CSMA/CD}

The mathematical model of CSMA/CD protocol is based on the following assumptions [5]:

1- Packet size is fixed.

2- Channel capacity is obtained by considering an infinite population model.

3- All devices collectively form an independent Poisson source.

4- Average retransmission delay is arbitrarily large.

5- The rate of devices becoming ready during a slot is $(\mathrm{k})$.

6- The transmission time of a packet (in slots) is equal to (T).

7- A transmission period (successful or unsuccessful) followed by an idle period is called a cycle as shown in Figure (2.4).with the infinite population assumption, all cycles are statistically identical. Figure (2.4a) shows a successful cycle which consists of a (Busy) period with length $(\boldsymbol{B}=\boldsymbol{T}+\mathbf{1})$ slots and Idle period with length equal $(I)$ slots; while Figure (2.4b) shows unsuccessful cycle which consists of collision period of length $(\boldsymbol{U}=\boldsymbol{\tau}+\mathbf{1})$ slots and Idle period with length equal $(I)$ slots.

8- The slot duration is equal to 1 (it is equal to the maximum propagation delay normalized with respect to its length).

9- There is a packet ready for transmission at the beginning of any cycle.

The Poisson distribution equation is given by:

$$
p_{j}(j)=\frac{(\lambda T)^{j} e^{-\lambda T}}{j !} \quad ; \quad j=(0,1,2,3 \ldots . .) \quad---(1)
$$

$$
p_{0}(0)
$$

Where:

$\lambda$ is aggregate mean packet generation rate (packet/Sec).
$\mathrm{T}$ is transmission time.

The packet of any cycle is considered to be successfully transmitted if there is no other packet to be arrived during the propagation delay (following the beginning of the packet transmission). The probability that there is only one packet in a cycle is given by

$$
p_{1}(1)=(\lambda T) e^{-\lambda T} \quad ; \quad J=1
$$

The probability of more than one packet existing in a cycle is given by

$p_{c}=\left(1-(\lambda T) e^{-\lambda T}\right) \quad---(2)$

The probability of successful transmission is defined by the following relationship:

$$
\begin{gathered}
p_{s}=\frac{p_{1}}{p_{c}}=k e^{-k} /\left(1-k e^{-k}\right) \quad---(3) \\
\text { Where } \quad k=\lambda \mathrm{T}
\end{gathered}
$$

The average Idle period is given by:

$$
\bar{I}=e^{-k} /\left(1-e^{-k}\right) \quad---(4)
$$

The average transmission period is

$$
\bar{B}=p_{s} T+\left(1-p_{s}\right) \tau+1 \quad---(5)
$$

The average length of a cycle is given by

$$
\bar{C}=\bar{B}+\bar{I}
$$

Finally, the throughput is given by:

$$
\begin{aligned}
& S=\frac{p_{S} T}{\bar{B}+\bar{I}}=\frac{T k e^{-k} /\left(1-e^{-k}\right)}{T k e^{-k} /\left(1-e^{-k}\right)+\left(1-k e^{-k} /\left(1-e^{-k}\right)\right) \tau+1+e^{-k} /\left(1-e^{-k}\right)} \\
& =\frac{T k e^{-k}}{T k e^{-k}+\left(1-e^{-k}-k e^{-k}\right) \tau+1} \quad---(6)
\end{aligned}
$$

Maximum channel capacity can be obtained by maximizing $\mathrm{S}$ with respect to $\mathrm{k}$.

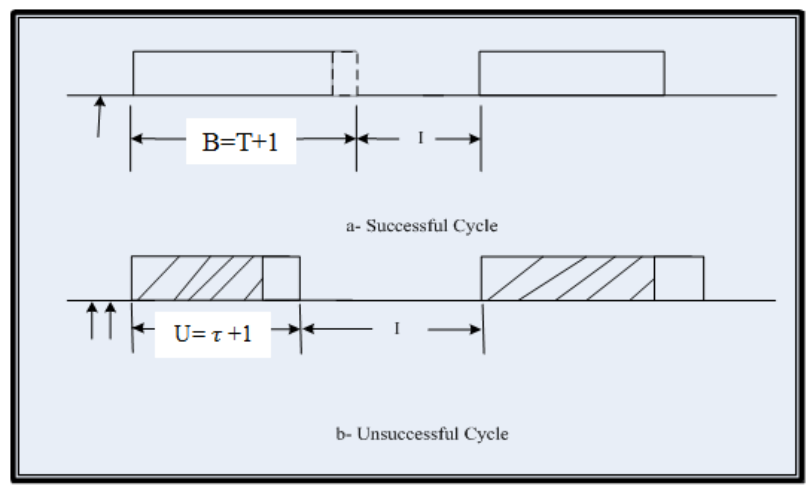

Figure (3) Transmission and Idle periods in slotted non persistent CSMA/CD

\section{DESIGN OF MAC DEVICE}

A network interface card can be represented from the simulation point of view by the block diagram shown in figure 
(4). The design of the NIC is based on the work shown in reference [6]. The units which constitute the NIC are as follows: the other is a copy of the transmitted packet. The details of this unit are as follows $[6,7]$ :

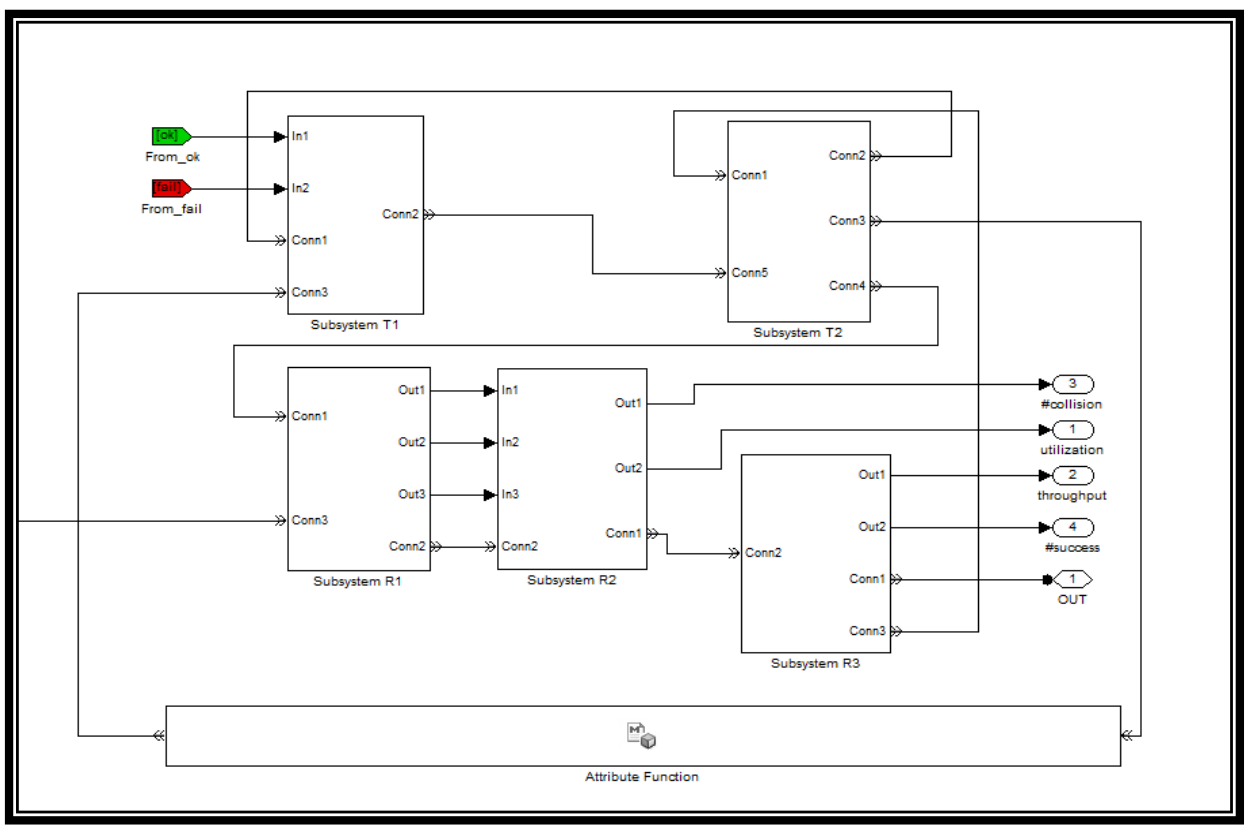

Figure (4) Network Interface Card block diagram

\subsection{Packet Generator}

This unit provides the necessary packets to test the performance of a given NIC. Figure (5) shows how Simevent tools are applied to generate the transmitted packets with the required Data rate and length (as it is generated in the application Layer).

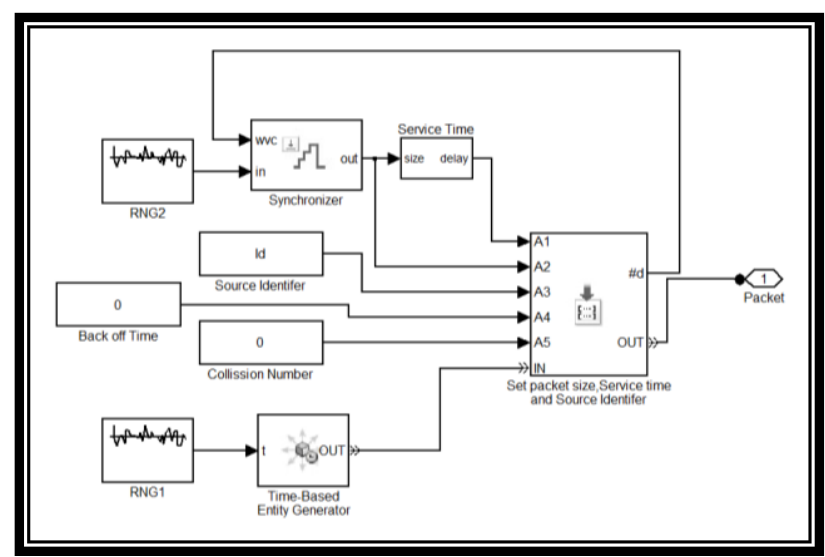

Figure (5) block diagram of the packet generator using Simevent technique

\subsection{NIC Transmitter Unit}

Figure (6) shows a block diagram of this unit, it has two input events and three input signals, Packet refers to the event that come from the upper layers, Retransmit event indicates that the already transmitted packet is subjected to collision which is to be retransmitted again, the successful signal indicates that the packet is transmitted successfully, while the Fail signal indicates that the transmitted packet is failed. The signal "chs" refers to channel state. The output of this unit shows two events one for the channel (transmit packet) and

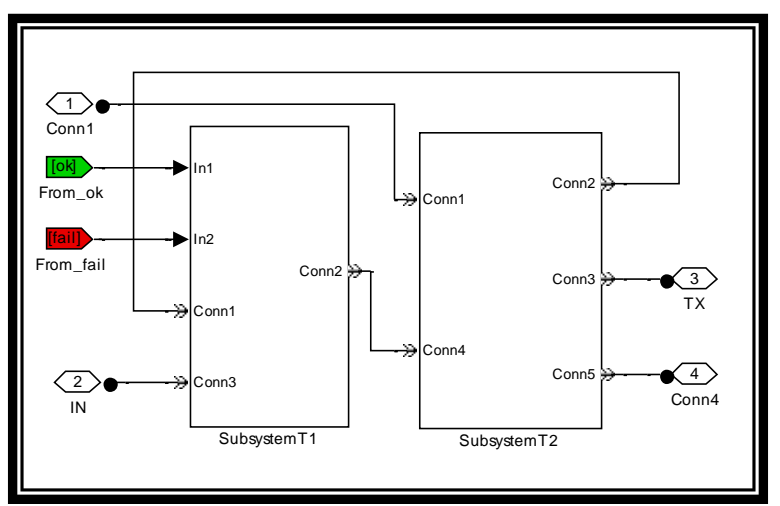

Figure (6) Transmitter Unit

a- SubsystemT1 Unit:

It consists of transmission buffer block which is responsible for receiving data from upper layer and queuing it in a fifo buffer, transmission control block removes the data from the buffer when the successful or drop signals are received, the source address block assign the MAC address (it is unique for each host) to the host, the Set Attribute block inserts this Mac address to the Data Segment to generate a packet, Start timer block is devoted to calculate delay time, The path combiner block receive either a new generated packet or a retransmitted packet at a given time, the output of this unit is connected to the input of the Single Server block, it is obvious that this unit organizes the arrival of the different packet to the Single Server block which transmits the packet after a period of time equal to the calculated Back off Time. Figure (7) shows the details of the subsystemT1 unit.

b- SubsystemT2 Unit: 
Figure (8) shows the details of this subsystems, which is consist of the following units:
3- Packet Transmitter block: The function of this block is to make two copies, the first one is inserted into the channel while the second one is forwarded to the receiver (to compare it with the received data from channel).

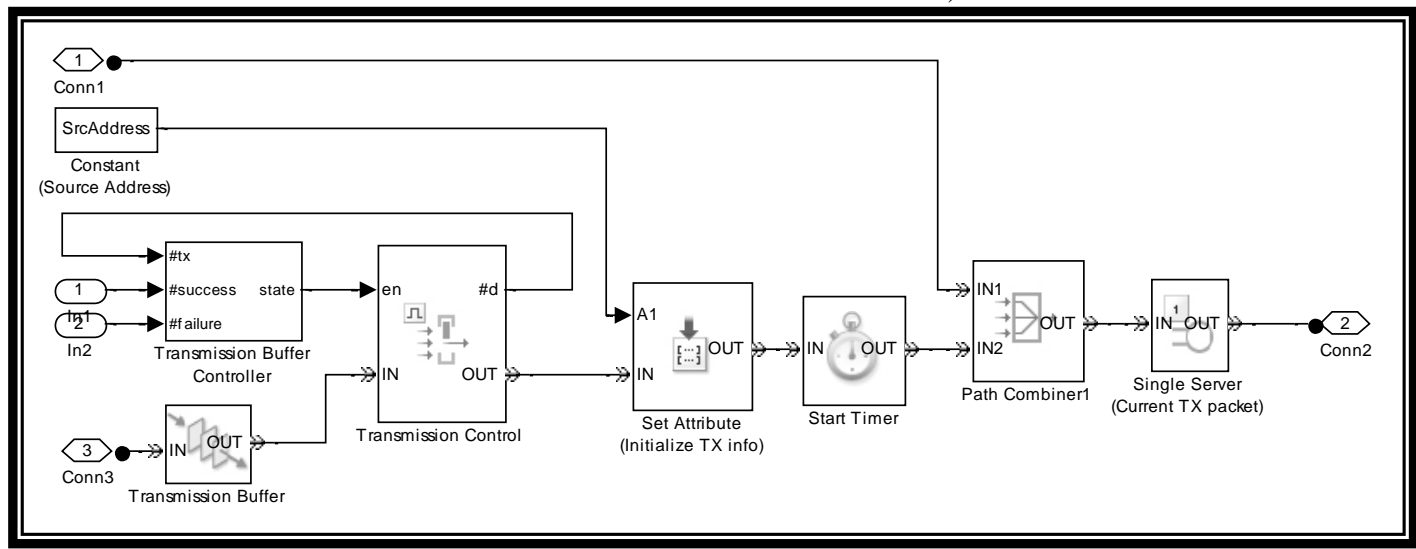

Figure (7) the details of subsystemT1 unit

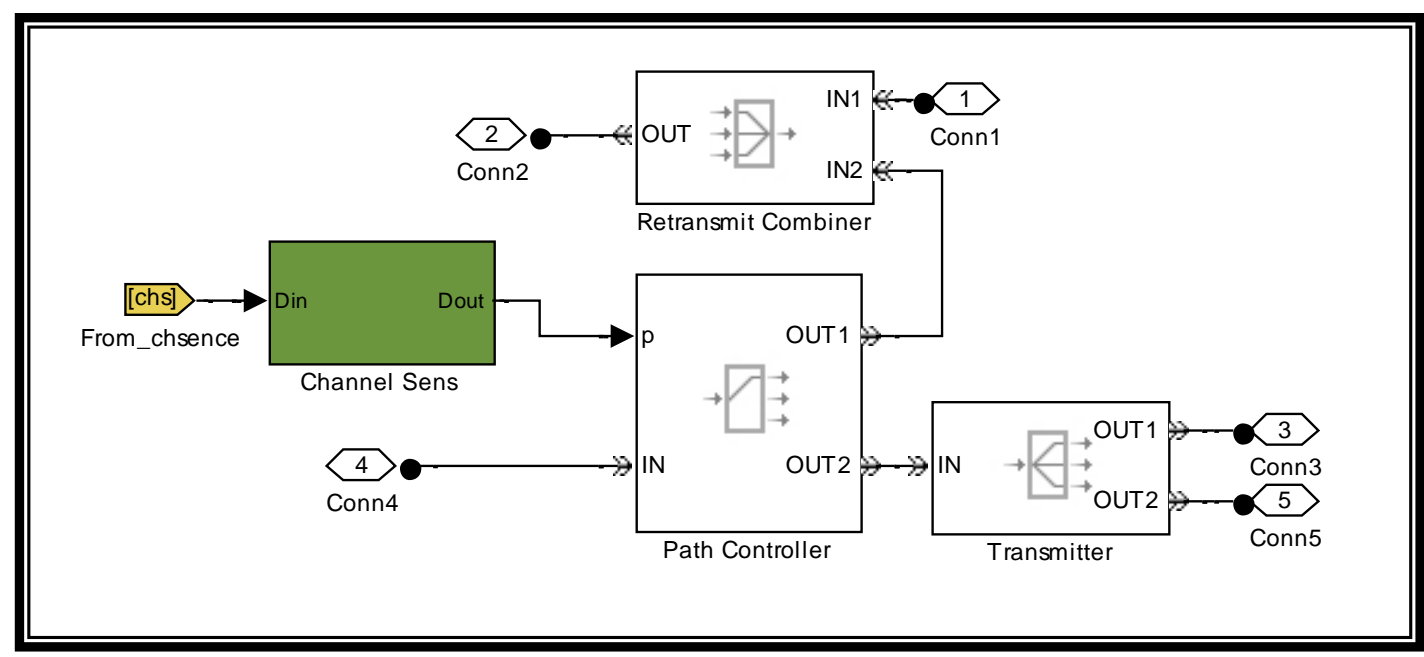

Figure (8) the details of subsystemT2 unit

1- Channel Sense Block: This block provides the sensing function of the channel, it is bounded by the value of the signal (chs) as follows:-

1- If chs $>0$ and Dout $=2$ then the channel is idle.

2- If chs $<=0$ and Dout $=1$ then the channel is busy.

Figure (9) shows the details of the channel sense block

2- Path Controller Block: This block is represented by a switch (using Simevents tools), it has two outputs, the first one (Out1) is connected to the Retransmit Combiner block and the second output is connected to the Packet transmitter block, the output of the channel sense block Dout is connected to the input "P" to control the function of this block. It controls the switch as follows:-

If "Dout" $=1$, then the Retransmitted packet will pass from "out1" to the input of Retransmit Combiner Block.

If "Dout" = 2, then the new packet will pass from "out2" to the input of the Path Transmit Block.
During the transmission of a packet, the subsystem A waits the two other signals (successful) signal (if the packet is successful) or (fail) signal (if the packet is failed).

4- Retransmit Combiner Block: This block is represented by Path Combiner Simevents Block; it receives either a collided packet or a new generated packet, (in the case that the channel is busy) and forwarded it to the input of the subsystem A unit.

\subsection{NIC Receiver Unit}

It consists of three Subsystems; SubsystemR1, SubsystemR2 and SubsystemR3 as shown in Figure (10). There are two input events, the first one is $\mathrm{Rx}$ which represent the packet received 
transmission while Out2 refers to the channel state in case of collisions. Figure (11) shows the details of this Unit.

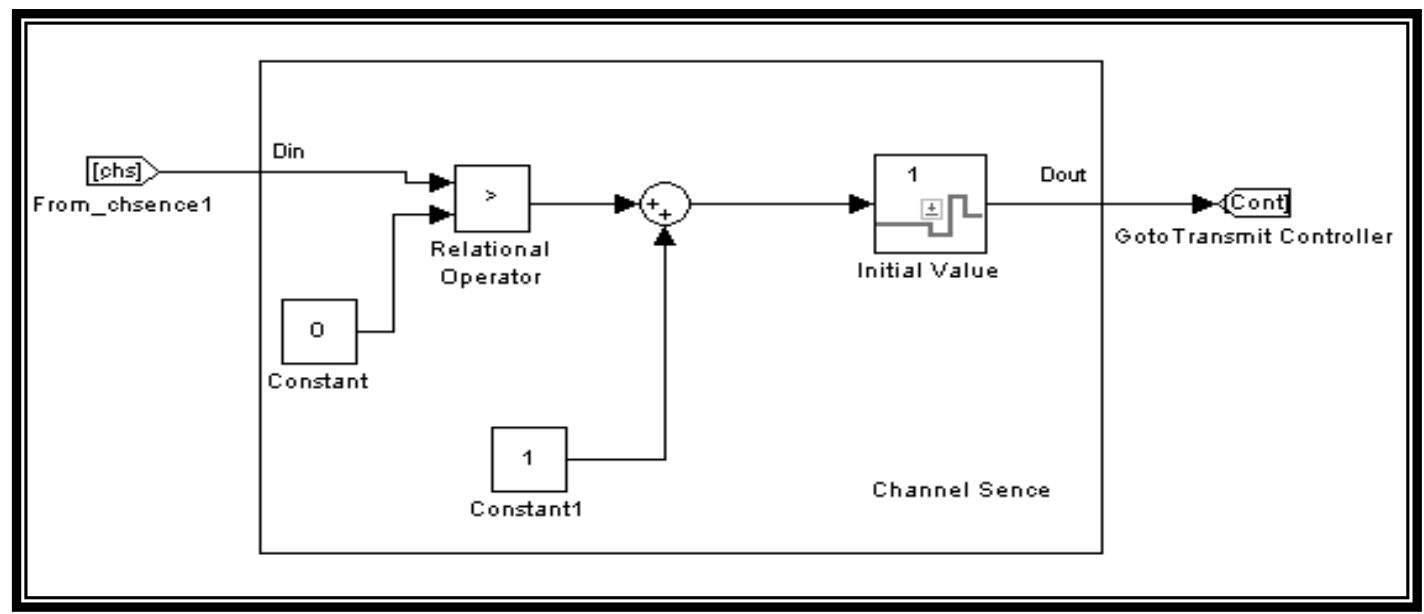

Figure (9) the details of channel sense block diagram

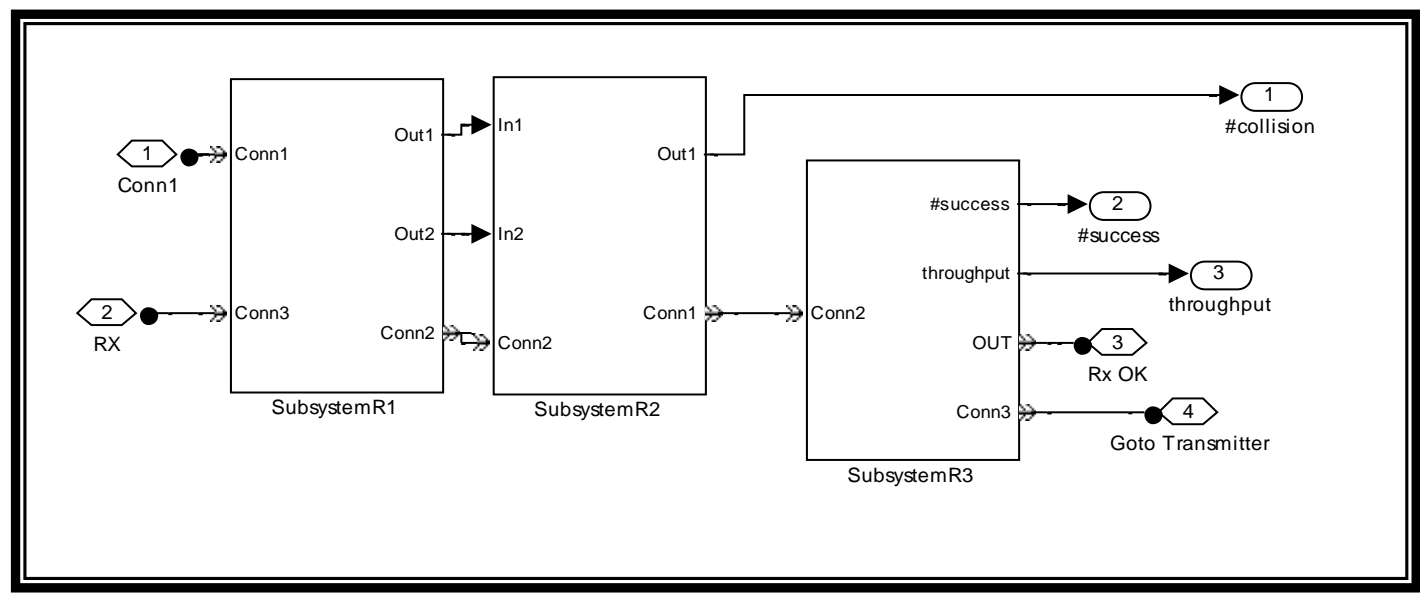

Figure (10) the receiver unit

from the Channel and the second one is the copy of the transmitted packet, also there are two output events, the first one is "Rx OK" and the second one is "Goto_transmite" refer to a collided packet which must be returned to the transmitter to retransmit it. There are other signals like "Goto chsense1", "Goto_ok", "Goto_fail" which are connected to the transmitter unit, the signals "\#success", "\#collision" are used to calculate the number of successful and collided packets, "transmit" and "throughput" are used to calculate the channel throughput. The Receiver Unit consists of the following units:

a- SubsystemR1:

It receives the packet from the output of the transmitter unit (Conn1) and receives a copy of this packet from the channel (Conn3) then sends the packet to the infinite server to delay it for a time equal to the size of the packet in bits divided by the bit rate of the channel (10 to power 7 for coaxial cable) this time is needed to transmit the packet through the channel, the output (\#n) represents the number of packets in the server, it is connected to the Chsense block to check the channel state, Out1 (chs) represents the channel state in case of successful b- SubsystemR2:

As shown in Figure (12) subsystemR2 is consist of the following blocks:

1- Packet state block: The function of this block is represented by the Set Attribute Block using Simevents tools; it is responsible about the addition of the information about the number of collisions (Out2) of the packet, to the data which is arrived from subsystem $B$.

2- Data extractor block: The function of this block can be represented by Get Attribute Block using Simevents tools; it extracts the source address, number of collisions and collision state from the data then this information should be send to the inputs of the back off controller unit, while the original data should pass to the input of the buffer.

3- Back off controller unit: The function of this block can be represented by the State Flow diagram shown in figure (13), the back off time is calculated from the back off algorithm, the total number of collisions can be computed too, also the new number of collisions of this packet can be checked. if it is greater than 15 then the packet will be dropped else it will be retransmitted after 
the collapse of the back off time, number of failed packets should be calculated too, finally the path (rout) of the output can be found.

4- Buffer Block: It can be represented by an infinite server, with a service time equal to zero; it receives the data from data extractor block and pass it to the attachment block.

5- Attachment block: The new values of the attributes will be assigning to the data. These attributes are calculated in the Back off controller unit (Path of the output (rout), new number of collisions of this packet and the back off time).

6- Output switch block: It is a four output switch; it passes the data through one of the four output ports depending upon the value of the path which is included in the attribute of the input data.

\section{SIMULATION OF ETHERNET BASEDON THE NIC:}

To validate the performance of the proposed Ethernet NIC (which is based on Simevent), a 10 base T Ethernet architecture is suggested and simulated using simevent tools. This architecture is included in the 802.3 standard. It is a hub based, and uses UTP as a transmission medium over a (100) $m$ length and (100) Mbps network bandwidth. Figure (15) shows the 10BaseT architecture block diagram. The simulation is based on the following assumptions:

1- UTP Cable (10BaseT).

2- Four computers.

3- Four Network Interface Cards of the proposed type.

4- HUB

5- The distance between any computer and HUB is equal to $(100 \mathrm{~m}$

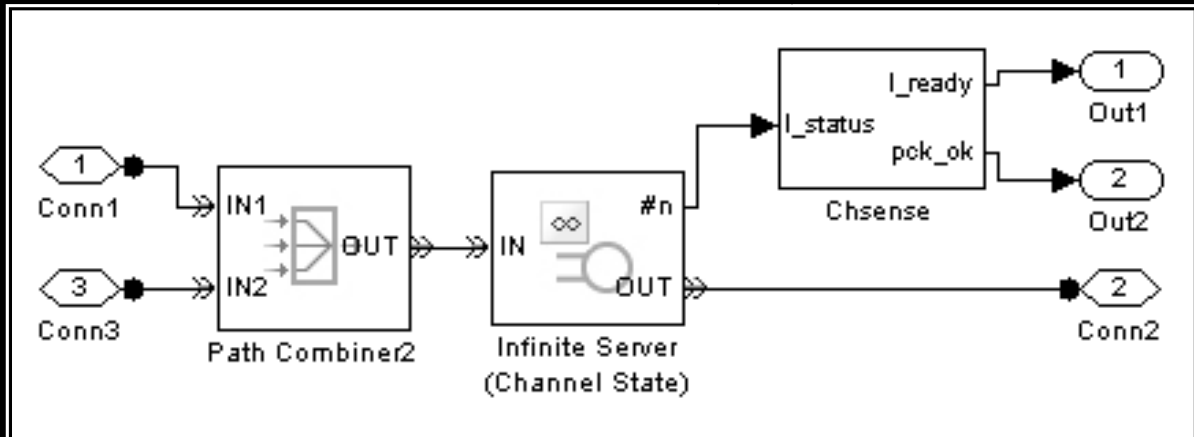

Figure (11) SubsystemR1 block diagram

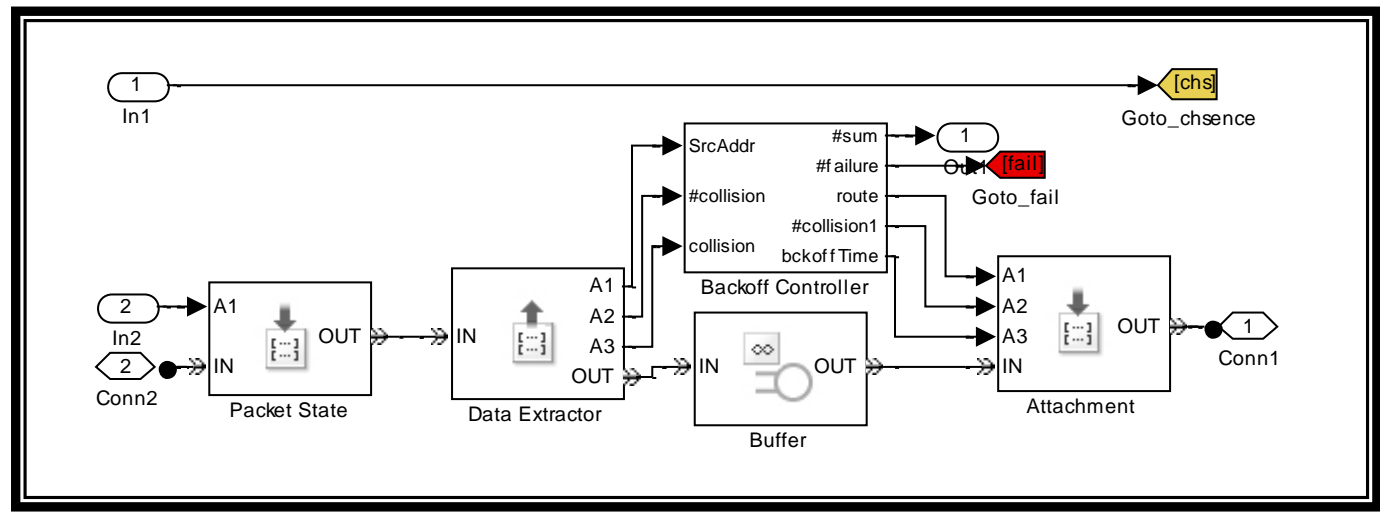

Figure (12) the details of SubsystemR2

c- SubsystemR3:

As shown in Figure (14) this subsystem is consist of the following blocks:

1- Entity sink unit: It receives the data and removes it from the channel if the transmission is successful or dropped it if the number of retransmissions exceeds (15) trails.

2- Read Timer unit: This block read the timer which represents the delay time.
The different blocks of figure (15) can be simulated as follows:

a-Computer representation unit:

Figure (16) shows the block diagram of the computer representation unit. The role of the upper layers from the simulations point of view is to generate packets according to the required bit rate and packet length, and to receive packets from other computers through the MAC Sublayer (the physical layer is implicitly included) 
b-HUB unit: Figure (17) shows the block diagram of four port HUB model; it consists of eight path combiner blocks; each port is represented by two path combiner block. The first one is considered as the input of the port and the second one represents the output of the port. Computers can be interconnected through HUB ports; it is worth to mention that each computer is connected to a single port of the HUB through a UTP cable. c- UTP cable: it is represented by two infinite servers. Figure (18) shows the block diagram of the UTP cable. The bandwidth will equal to (100Mbps) so this will change the service time which is calculated in the packets generator system using equation (7).

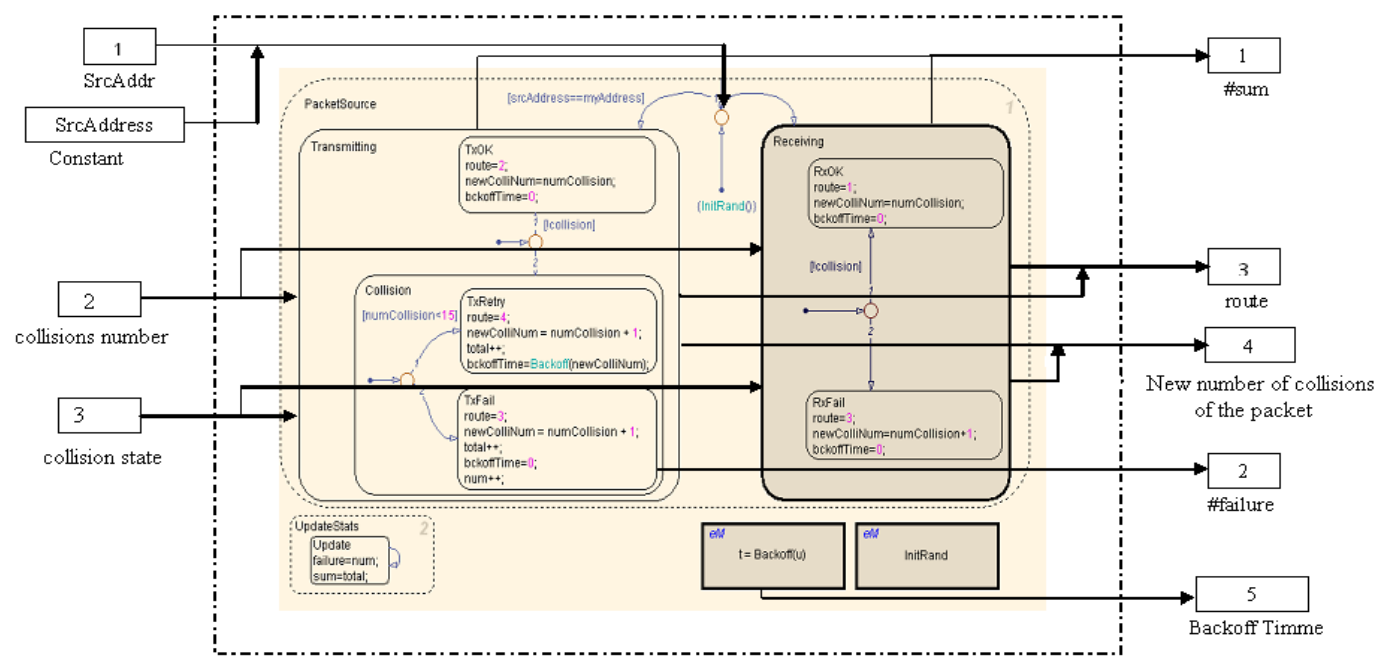

Figure (13) Back off Controller state flow block diagram

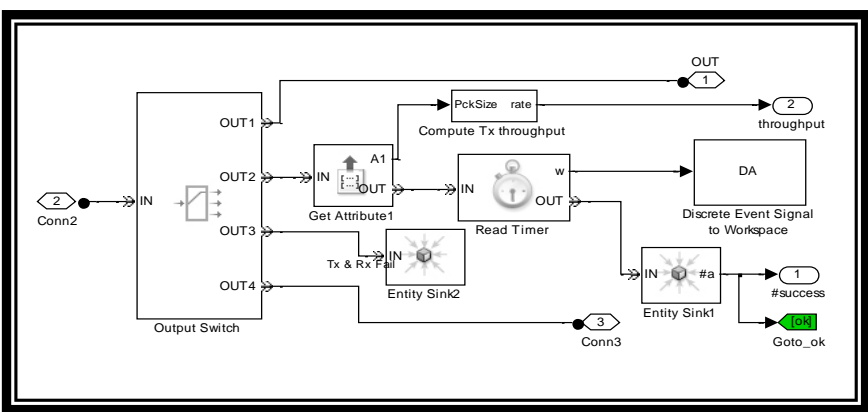

Figure (14) the details of SubsystemR3

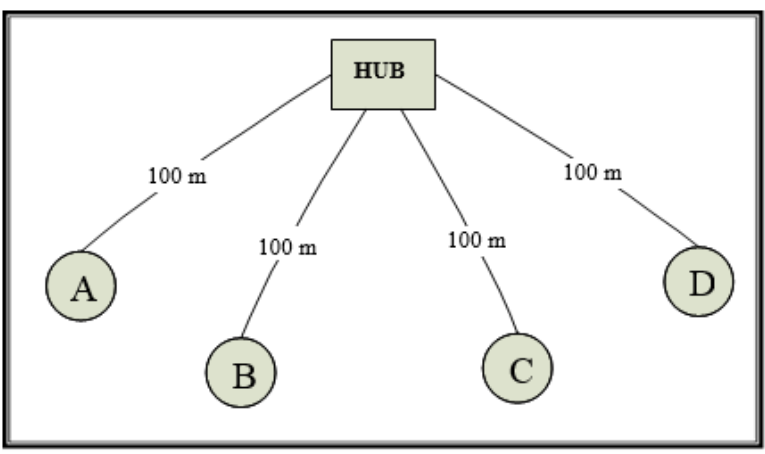

Figure (15) 10BaseT architecture block diagram

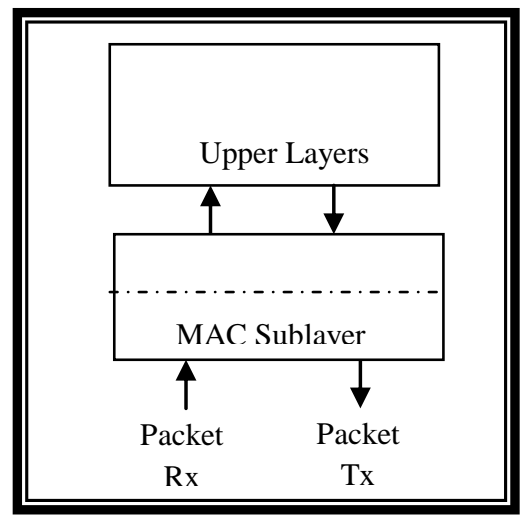

Figure (16) computer representation block diagram

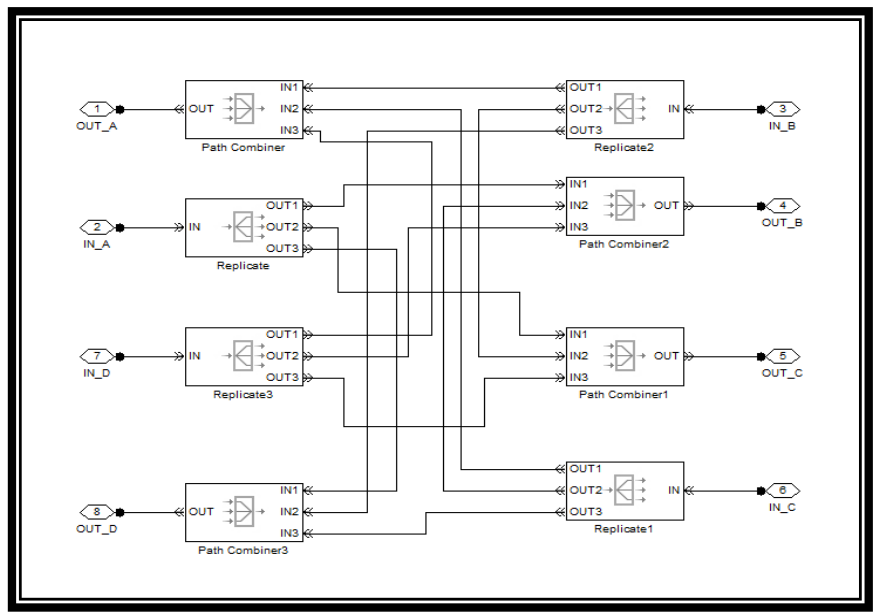

Figure (17) Four port HUB block diagram 
$t_{s}=\frac{\text { packet length }(\text { bit })}{B W(b p s)}---(7)$

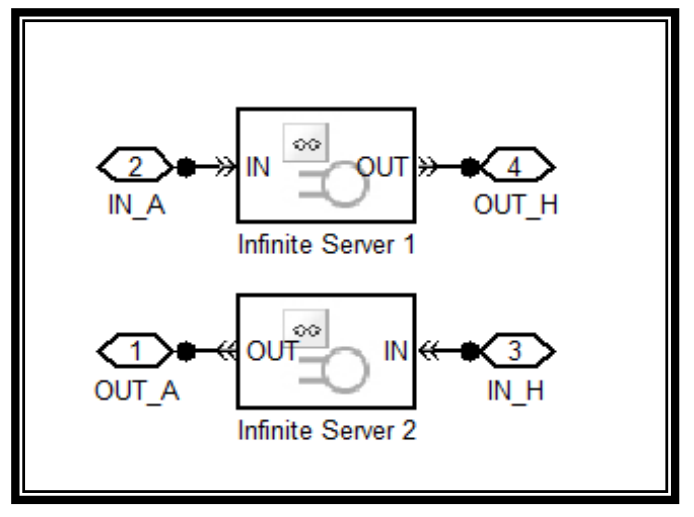

Figure (18) UTP cable block diagram

Finally, Figure (19) shows a complete simevent equivalent block diagram of the network which is given in figure (15).

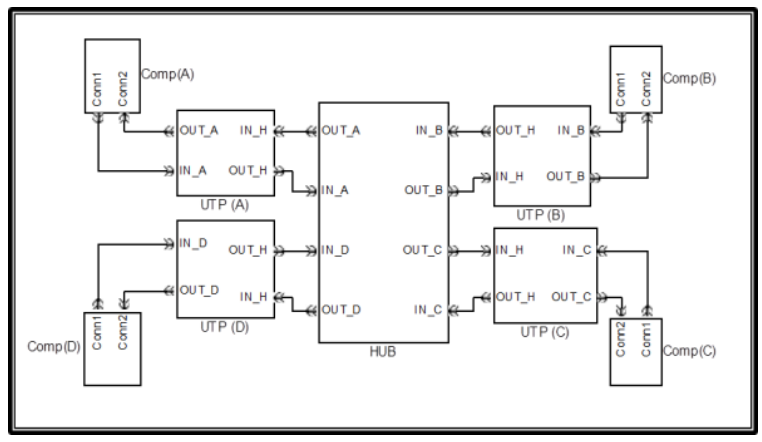

Figure (19) the simulation model of UTP architecture

\section{SIMULATION OF ETHERNET USING SIMULINK:}

To validate the performance of the Ethernet given in figure (15), Simulink software is used as an alternative approach, the setting of the Simulink parameters takes into account the same model assumptions which are used to build the simevent equivalent block diagram of Ethernet. Figure (20) shows the block diagram of Ethernet from the Simulink simulation point of view.

\section{SIMULATION OF ETHERNET USING OPNET:}

Again to validate the performance of the Ethernet given in figure (15), OPNET software is used as an alternative approach, the setting of the OPNET parameters takes into account the some model assumptions which are used to build the simevent equivalent block diagram of Ethernet. Figure (21) shows the block diagram of Ethernet from the OPNET simulation point of view [8].

\section{SIMULATION RESULTS:}

The simulation results being obtained are based on the following assumptions:

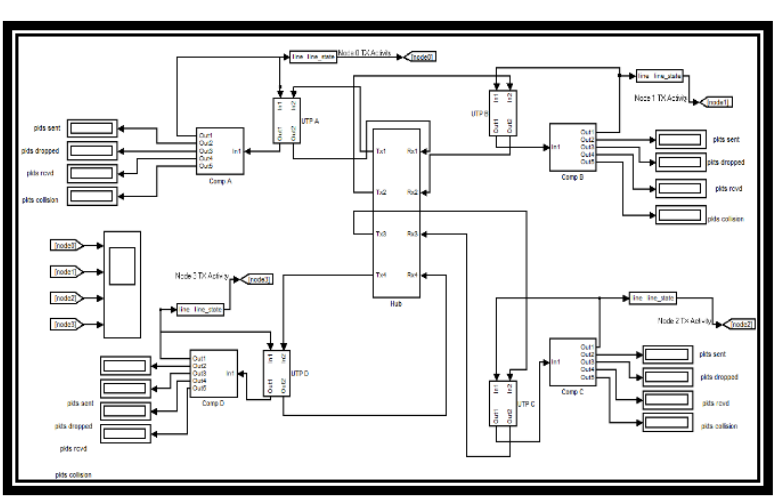

Figure (20) Star topology network (10BaseT) Architecture using Simulink program

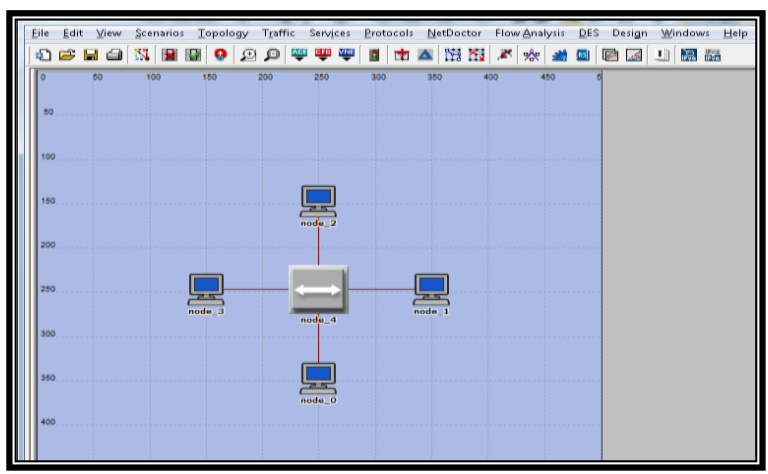

Figure (21) Star topology network (10BaseT) Architecture using OPNET Modeler program

a- Packet generation rate $=(10$ to 250$)$ packet $/ \mathrm{sec}$.

b- $\quad$ Packet size $=1000$ byte.

c- The time of simulation $=80000 /$ packet generation rate.

d- The normalized throughput is calculated using the following formula.

$$
S=\frac{\text { No. of Successful packets }}{\text { Total No.of packets }}
$$

e- Any packet will be dropped after 15 retransmission trials.

The simulation results which are obtained are as follows:-

1- The normalized throughput performance as a function of the packet rate generation is shown in Figure (22).

2- The delay performance as a function of packet rate generation is shown in Figure (23).

3- The number of successful Packets as a function of packet rate generation is shown in Figure (24). 


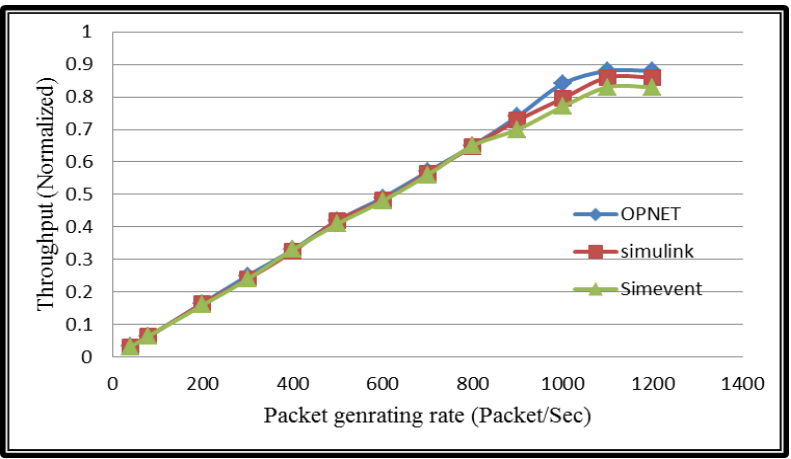

Figure (22) Throughput performance as a function of packet rate generation

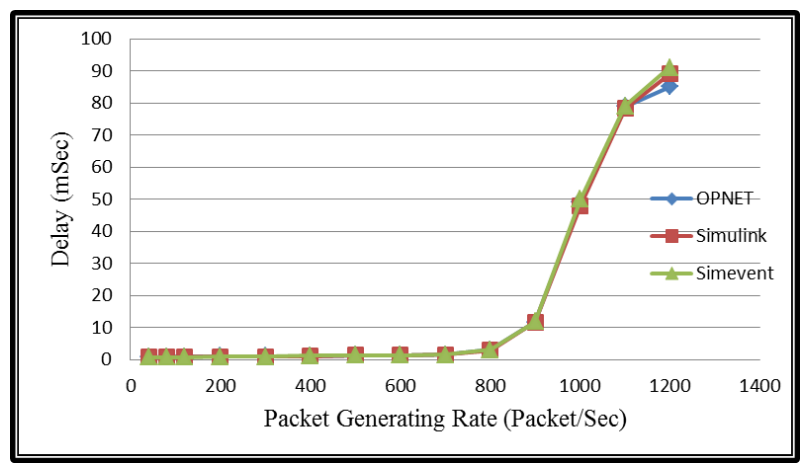

Figure (23) Delay performance as a function of packet generation rate for $10 B a s e T$ architecture

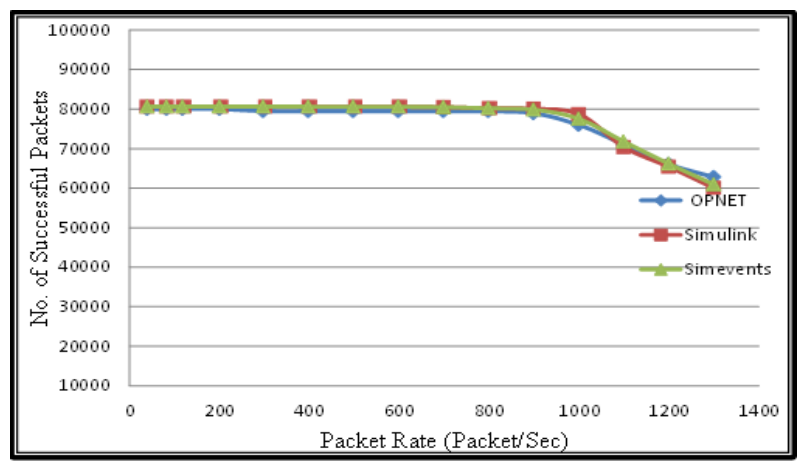

Figure (24) Number of successful Packet as a function of packet generation rate for 10BaseT architecture

\section{CONCLUSION}

It is obvious that the introduction of Simevent tools in the design of network interface card will provide an efficient way of selecting the parameters of the network, it is more realistic than OPNET software due to the fact that each Simevent unit can be adjusted separately the network constructed with Simevent tools behaves like actual Ethernet which means that the possibility of modifying any unit can be achieved easily regardless the condition of other units.

The results of throughput and delay performances using Simevent, Simulink and OPNET approaches are compared and show an excellent agreement with each other especially when the overall packet rate is less than (1000).

\section{REFERENCES}

[1] Deon Reynders, Edwin Wright, "Practical TCP/IP and Ethernet Networking”, IDC Technologies, 2003.

[2] Sharam Hekmat, "Communication Networks", PragSoft Corporation, www.pragsoft.com., Copyright (C) 2005 PragSoft.

[3] Martin H. Davis, Jr., iCore Technologies, Inc., Dayton, Ohio; "AN INTELLIGENT NETWORK INTERFACE CARD”, IEEE, 1999.

[4] Andrea Bianco, Robert Birke, Gianluca Botto, Marcello Chiaberge, Jorge M. Finochietto, Giulio Galante,Marco Mellia, Fabio Neri, Michele etracca; "Boosting the Performance of PC-based Software Routers with FPGAenhanced Network Interface Cards”, IEEE 2006.

[5] Fouad A. Tobagi and V. Bruce Hunt, "Performance Analysis of Carrier Sense Multiple Access With Collision Detection", Computer Networks, North-Holand publishing company, Volume 4, Issue 5, pp 245-259, October-November 1980.

[6] "Ethernet Local Area Network", MATLAB Information Center available on: www.mathworks.com/trademarks.

[7] Tanenbaum, Andrew S., "Computer Networks", Fourth Ed. , Upper Saddle River, N.J., Prentice hall PTR 2002.

[8] www.opnet.com, “OPNET Modeler 11.5”, 2005.

[9] B. A. Forouzan, S. C. Fegan, "TCP/IP Protocol Suite", Second ed., New York, McGraw-Hill, 2003. 